\title{
Open relations and collectionwise normality
}

\author{
by \\ Valentin GUTEV \\ Presented by Czesław BESSAGA
}

Dedicated to the memory of Stoyan Nedev

\begin{abstract}
Summary. Using the framework of discrete-valued relations, we give a simple proof of a theorem obtained by Stoyan Nedev. This theorem provides a generalisation of an element in the proof of Dowker's extension theorem, which is essential for constructing continuous selections of set-valued mappings defined on collectionwise normal spaces. Using this relationship, we also give a simple proof of the Dowker's theorem.
\end{abstract}

1. Introduction. A $T_{1}$-space $X$ is collectionwise normal (Bing [1]) if for every discrete collection $\mathscr{D}$ of subsets of $X$ there exists a discrete collection $\left\{U_{D}: D \in \mathscr{D}\right\}$ of open subsets of $X$ such that $D \subset U_{D}$ for all $D \in \mathscr{D}$. According to this definition, every collectionwise normal space is Hausdorff, and also normal. In the same paper (see [1, Examples $\mathrm{G}$ and H]), Bing described an example of a normal space which is not collectionwise normal, now known as Bing's example. He also proved that full normality (i.e. paracompactness) implies collectionwise normality but not conversely [1, Theorem 12]. Since the closures of the elements of a discrete collection form a discrete collection too, a $T_{1}$-space $X$ is collectionwise normal if for every discrete collection $\mathscr{D}$ of closed subsets of $X$ there exists a discrete collection $\left\{U_{D}: D \in \mathscr{D}\right\}$ of open subsets of $X$ such that $D \subset U_{D}$ for all $D \in \mathscr{D}$. In the presence of normality, this can be further relaxed to requiring $\left\{U_{D}: D \in \mathscr{D}\right\}$ to be only pairwise disjoint.

Collectionwise normality is a natural generalisation of normality. Indeed, if $X$ has this property with respect to discrete families $\mathscr{D}$ of cardinality at

2010 Mathematics Subject Classification: 54C60, 54D15, 54B20, 54C20.

Key words and phrases: relation, set-valued mapping, collectionwise normality, extension. Received 15 January 2017; revised 18 April 2017.

Published online 8 June 2017. 
most $\tau$, then it is called $\tau$-collectionwise normal. Thus, $X$ is normal iff it is 2-collectionwise normal (or, more generally, $n$-collectionwise normal for every finite $n \geq 2$ ). In fact, a space $X$ is normal iff it is $\omega$-collectionwise normal, which follows easily from Urysohn's characterisation of normality. On the other hand, for every cardinal $\tau$ there exists a $\tau$-collectionwise normal space which is not $\tau^{+}$-collectionwise normal [11], where the cardinal $\tau^{+}$is the immediate successor of $\tau$.

The following theorem was proved by St. Nedev [9] (see also [10]) and is commonly called Nedev's lemma. The theorem provides a paracompact-like property of collectionwise normality. In this theorem, a family $\mathscr{V}$ of subsets of $X$ is said to refine another family $\mathscr{U}$ if each element of $\mathscr{V}$ is a subset of some element of $\mathscr{U}$.

TheOREm 1.1. Let $X$ be a collectionwise normal space, $A \subset X$ a closed subset, and $\mathscr{U}$ an open (in $X$ ) cover of $A$ which is point-finite in $A$. Then there exists a locally finite and open (in $X)$ cover of $A$ which refines $\mathscr{U}$.

For a locally finite cover of $A$, Theorem 1.1 was proved by Dowker [2]. In another special case when $A=X$, this theorem was obtained independently by Michael [7] and Nagami [8].

The purpose of this paper is to give a simple proof of Theorem 1.1 based on the framework of relations. Here is briefly the idea. If $\{\mathscr{U}(y): y \in Y\}$ is an indexed family of subsets of $X$, then it represents a map $\mathscr{U}$ from $Y$ to the subsets of $X$. This map is uniquely determined by the relation $\mathscr{U}=\{\langle y, x\rangle$ : $x \in \mathscr{U}(y)\}$, i.e. by its graph. In the next section, we discuss the advantage of considering the inverse relation $\mathscr{U}^{-1}=\{\langle x, y\rangle:\langle y, x\rangle \in \mathscr{U}\} \subset X \times Y$. It follows at once that the open families in $X$ indexed by the elements of a discrete space $Y$ are precisely the open subsets of $X \times Y$ (Proposition 2.1), i.e. the open relations in $X \times Y$. In other words, the open families in $X$ indexed by a discrete space $Y$ are nothing other than the topology of the product space $X \times Y$. This will be found very useful because for a discrete $Y$, the product $X \times Y$ is normal whenever so is $X$.

Each relation $\Phi \subset X \times Y$ represents a set-valued mapping from the domain $\operatorname{dom}(\Phi)$ of $\Phi$ to the subsets of $Y$. In the framework of set-valued mappings, special families of subsets of $X$ indexed by a discrete space $Y$ have also natural interpretations. Thus, the locally finite families $\Phi \subset X \times Y$ are the well-known subcontinuous set-valued mappings (see Proposition 2.1](c)). The discrete collections of subsets of $X$ are the usual (partial) continuous maps $\Phi: \operatorname{dom}(\Phi) \rightarrow Y$ (Proposition 3.1), while the point-finite families are precisely the finite-valued mappings, i.e. the finite-valued relations in $X \times Y$. In Section 3, we show that each open finite-valued relation determines a sequence of partial continuous maps in the "Vietoris hyperspace" of its range (Proposition 3.4). Things are culminating in Section 4, where we provide 
an interpretation of collectionwise normality in terms of extending partial continuous maps in discrete spaces (Proposition 4.1), and next we illustrate our proof of Theorem 1.1 (see Theorem 4.2).

Let us remark that Theorem 1.1 is crucial in generalising Dowker's extension theorem [2] in terms of continuous selections of set-valued mappings. In the last Section 5, using a natural relationship between extensions and selections for collectionwise normal spaces [5], we also provide a simple proof of this theorem of Dowker.

2. Open subcontinuous relations. A relation $\Phi$ from $X$ to $Y$ is a synonym of a subset $\Phi \subset X \times Y$. In this interpretation, $\Phi(x)=\{y \in Y$ : $\langle x, y\rangle \in \Phi\}$ is the image of $x \in X$ by $\Phi$, and $\Phi^{-1}(y)=\{x \in X:\langle x, y\rangle \in \Phi\}$ is the preimage of $y \in Y$. More generally, $\Phi[A]=\bigcup_{x \in A} \Phi(x)$ is the image of a subset $A \subset X$ by $\Phi$, and $\Phi^{-1}[B]=\bigcup_{y \in B} \Phi^{-1}(y)$ is the preimage of $B \subset Y$. In fact, in terms of the inverse relation $\Phi^{-1}=\{\langle y, x\rangle:\langle x, y\rangle \in \Phi\}$, preimages by $\Phi$ are merely images by $\Phi^{-1}$. Finally, to each relation $\Phi \subset X \times Y$, we will associate the complement relation $X \times Y \backslash \Phi$ denoted for simplicity by $\Phi^{\mathrm{c}}=X \times Y \backslash \Phi$.

The domain of a relation $\Phi \subset X \times Y$ is the set $\operatorname{dom}(\Phi)=\Phi^{-1}[Y]$. If $\operatorname{dom}(\Phi)=X$, we will simply write $\Phi: X \rightsquigarrow Y$, which is another way to indicate that $\Phi$ is a set-valued mapping from $X$ to the (nonempty) subsets of $Y$. Thus, each relation $\Phi \subset X \times Y$ represents the partial set-valued mapping $\Phi: \operatorname{dom}(\Phi) \rightsquigarrow Y$. In this regard, when clarity seems to demand it, we will write $\Phi: \operatorname{dom}(\Phi) \rightarrow Y$ to emphasise that $\Phi$ is a usual map from $\operatorname{dom}(\Phi)$ to $Y$ and will say that $\Phi \subset X \times Y$ is a partial map.

As stated in the Introduction, an indexed family $\{\mathscr{U}(y): y \in Y\}$ of subsets of a set $X$ is nothing other than the relation $\mathscr{U}=\{\langle y, x\rangle$ : $x \in \mathscr{U}(y)\}$. If $\mathscr{U}$ is also a cover of $X$, then $X=\bigcup_{y \in Y} \mathscr{U}(y)=\operatorname{dom}\left(\mathscr{U}^{-1}\right)$, and therefore $\mathscr{U}^{-1}: X \rightsquigarrow Y$. The converse is also true, and the inverse $\Phi^{-1}$ of each relation $\Phi \subset X \times Y$ represents the indexed family $\left\{\Phi^{-1}(y): y \in Y\right\}$ of subsets of $X$. Thus, the families of subsets of $X$ indexed by a set $Y$ are precisely the relations $\Phi \subset X \times Y$, whereas the covers are precisely the set-valued mappings $\Phi: X \rightsquigarrow Y$. Motivated by this, we will sometimes refer to a relation $\Phi \subset X \times Y$ as a $Y$-family in $X$ (respectively, $Y$-cover of $X$ ) to express properties of the family $\left\{\Phi^{-1}(y): y \in Y\right\}$. In fact, such properties are in good accord with properties of relations as the following simple observation asserts (see also [4, Propositions 3.1 and 3.4]).

Proposition 2.1. Let $X$ be a space, $Y$ a discrete space, and $\Phi \subset X \times Y$. Then:

(a) $\Phi$ is an open $Y$-family in $X$ if and only if $\Phi$ is an open relation.

(b) $\Phi$ is a closed $Y$-family in $X$ if and only if $\Phi$ is a closed relation. 
(c) $\Phi$ is a locally finite $Y$-family in $X$ if and only if each $p \in X$ is contained in an open set $V \subset X$ with $\Phi[V] \subset Y$ being a finite set.

Proof. Since $Y$ is discrete, each $\Phi^{-1}(y), y \in Y$, is open in $X$ precisely when $\Phi^{-1}(y) \times\{y\}$ is open in $X \times Y$, for every $y \in Y$. This is (a). To see (b) apply (a) to the complement relation $\Phi^{\mathrm{c}}$. Finally, (c) follows from the fact that $\Phi[V]=\left\{y \in Y: V \cap \Phi^{-1}(y) \neq \emptyset\right\}$ whenever $V \subset X$.

We shall say that a relation $\Phi \subset X \times Y$ is subcontinuous at $p \in X$ if for every open cover of $Y$, the point $p$ is contained in an open set $V \subset X$ such that $\Phi[V]$ is covered by finitely many members of this cover; and we say that $\Phi$ is subcontinuous if it is subcontinuous at each point of $\operatorname{dom}(\Phi)$. Originally, subcontinuity was defined for usual maps in [3]; subsequently, the property was extended to set-valued mappings in [12]. Our definition is adapted to set-valued mappings, and a relation $\Phi \subset X \times Y$ is subcontinuous if the corresponding set-valued mapping $\Phi: \operatorname{dom}(\Phi) \rightsquigarrow Y$ is subcontinuous. In our considerations, it will also make sense to consider a stronger property by saying that a relation $\Phi \subset X \times Y$ is strongly subcontinuous if $\Phi$ is subcontinuous at each point of $X$. Since each cover of $Y$ is refined by the singletons of $Y$, Proposition 2.1 asserts that a relation $\Phi \subset X \times Y$, with $Y$ being discrete, is strongly subcontinuous iff the $Y$-family represented by $\Phi$ is locally finite in $X$; and implies that $\Phi$ is subcontinuous iff this family is locally finite in itself, i.e. in $\bigcup_{y \in Y} \Phi^{-1}(y)=\operatorname{dom}(\Phi)$. Thus, in this setting, "strong subcontinuity" is a synonym for "locally finite in $X$ ".

It is evident that each subcontinuous relation with a closed domain is strongly subcontinuous. Based on this, we have the following natural construction of strongly subcontinuous relations.

Proposition 2.2. Let $X$ be a space, $Y$ a discrete space, and $\Phi \subset X \times Y$ a subcontinuous relation. Then the restricted relation $\Phi\lceil B=\Phi \cap B \times Y$ is strongly subcontinuous for every $B \subset X$ with $\bar{B} \subset \operatorname{dom}(\Phi)$.

Proof. At the points of $\bar{B}$, the subcontinuity of $\Phi \nmid B$ follows from that of $\Phi$ because $\bar{B} \subset \operatorname{dom}(\Phi)$ and $\Phi\lceil B \subset \Phi$. At the points of $X \backslash \bar{B}$, we simply have $(\Phi\lceil B)[X \backslash \bar{B}]=\emptyset$.

For spaces $X$ and $Y$, the collection $\mathscr{O}[X, Y]$ of all open relations in $X \times Y$ is the product topology on $X \times Y$. If $Y$ is discrete, according to Proposition 2.1. $\mathscr{O}[X, Y]$ is the collection of all open $Y$-families of $X$. This interpretation will be found very useful in dealing with locally finite open families in a normal space $X$, because in this case the product $X \times Y$ is itself a normal space, being a topological sum of normal spaces. Our considerations will be based on the simple observation that each countable open cover of a space $X$ has a locally finite refinement (see [6, Lemma 2.2]). To this end, let $\omega$ be the first infinite ordinal. To each relation $\Phi \subset X \times \omega$ we associate the epi-relation 
$\Phi_{\text {epi }} \subset X \times \omega$ defined by

$$
\Phi_{\text {epi }}=\{\langle x, k\rangle: n \leq k \text { for some }\langle x, n\rangle \in \Phi\} .
$$

If $\omega$ is replaced by $\mathbb{R}$, and $\Phi$ by a usual function $f: X \rightarrow \mathbb{R}$, the epi-relation $f_{\text {epi }}$ is just the epigraph of $f$. In our case, the epi-relations represent the increasing countable families in $X$. Namely, $\Phi \subset X \times \omega$ is an increasing $\omega$-family in $X$ if $\Phi^{-1}(n) \subset \Phi^{-1}(k)$, whenever $n \leq k<\omega$; equivalently, if $\Phi=\Phi_{\text {epi }}$. Furthermore, the epi-relation $\Phi_{\text {epi }}$ is open (closed) whenever so is $\Phi \subset X \times \omega$, because $\Phi_{\text {epi }}^{-1}(k)=\bigcup_{n<k} \Phi^{-1}(n), k<\omega$. Finally, to each relation $\Phi \subset X \times \omega$ we will also associate the relation

$$
\Phi^{\uparrow}=\{\langle x, k+1\rangle:\langle x, k\rangle \in \Phi\}
$$

obtained by shifting the values of $\Phi$ upwards. Evidently, $\left[\Phi_{\text {epi }}\right]^{\uparrow}=\left[\Phi^{\uparrow}\right]_{\text {epi }}$. Moreover, $\Phi_{\text {epi }}^{\uparrow}$ remains open [closed] provided so is $\Phi$.

In the next proposition, we consider $\omega$ as an ordinal space equipped with the open interval topology, or equivalently with the discrete topology.

Proposition 2.3. Let $X$ be a space, $\Phi \subset X \times \omega$ and $\left[\Phi_{\mathrm{epi}}^{\uparrow}\right]^{c}=X \times \omega \backslash \Phi_{\mathrm{epi}}^{\uparrow}$ be the complement relation of $\Phi_{\text {epi }}^{\uparrow}$. Then $\operatorname{dom}(\Phi) \subset \operatorname{dom}\left(\left[\Phi_{\mathrm{epi}}^{\uparrow}\right]^{\mathrm{c}}\right)$. Moreover, the restricted relation $\left[\Phi_{\mathrm{epi}}^{\uparrow}\right]^{\mathrm{c}}\lceil\operatorname{dom}(\Phi)$ is subcontinuous whenever $\Phi$ is open.

Proof. Take $p \in \operatorname{dom}(\Phi)$, and let $k \in \Phi(p)$ be the minimal element of $\Phi(p)$. Then $(\leftarrow, k]=\omega \backslash \Phi_{\mathrm{epi}}^{\uparrow}(p) \neq \emptyset$. If moreover $\Phi$ is open, then $V=\Phi^{-1}(k)$ is an open set with $p \in V$ and $\left[\Phi_{\text {epi }}^{\uparrow}\right]^{\mathrm{c}}[V] \subset(\leftarrow, k]$. Indeed, $x \in V$ implies $k \in \Phi(x)$, and consequently $[k+1, \rightarrow) \subset \Phi_{\mathrm{epi}}^{\uparrow}(x)$.

We now have the following crucial construction of open subcontinuous relations.

Proposition 2.4. Let $X$ be a normal space, $\Phi \subset X \times \omega$ an open relation, and $\Psi \subset X \times \omega$ a closed relation with $\Psi \subset \Phi$. Then $\Phi$ contains an open subcontinuous relation $\Omega \subset X \times \omega$ with $\operatorname{dom}(\Psi) \subset \operatorname{dom}(\Omega)$.

Proof. Since $X \times \omega$ is normal (because so is $X$ ), $\Psi \subset \varphi \subset \bar{\varphi}=\psi \subset \Phi$ for some open relation $\varphi \subset X \times \omega$. For convenience, let $U=\operatorname{dom}(\varphi)$, which is an open set because $\varphi \subset X \times Y$ is open (see Proposition 2.1). We are going to show that $\Omega=\Phi \cap\left[\psi_{\text {epi }}^{\uparrow}\right]^{c} \mid U$ is as required. Clearly, the relation $\Omega$ is open because $\Phi \backslash U$ is open and $\psi$ is closed. By Proposition 2.3, the relation $\left[\varphi_{\mathrm{epi}}^{\uparrow}\right]^{\mathrm{c}} \uparrow U=\left[\varphi_{\mathrm{epi}}^{\uparrow}\right]^{\mathrm{c}} \uparrow \operatorname{dom}(\varphi)$ is subcontinuous because $\varphi$ is open. Hence, so is $\Omega$ because $\Omega \subset\left[\psi_{\text {epi }}^{\uparrow}\right]^{c}\left|U \subset\left[\varphi_{\text {epi }}^{\uparrow}\right]^{c}\right| U$. Finally, using Proposition 2.3 once again, we also get

$$
\begin{aligned}
\operatorname{dom}(\Psi) \subset \operatorname{dom}(\Phi)\lceil U & =\operatorname{dom}\left(\Phi \cap\left[\Phi_{\mathrm{epi}}^{\uparrow}\right]^{\mathrm{c}}\right)\lceil U \\
& \subset \operatorname{dom}\left(\Phi \cap\left[\psi_{\mathrm{epi}}^{\uparrow}\right]^{\mathrm{c}}\right)\lceil U \\
& =\operatorname{dom}(\Omega) .
\end{aligned}
$$


Applying this to a sequence of relations, we get the following consequence.

Corollary 2.5. Let $X$ be a normal space, $Y$ a discrete space, $\left\{\Omega_{n}\right\}_{n<\omega}$ a sequence of open subcontinuous relations $\Omega_{n} \subset X \times Y$, and $\left\{F_{n}\right\}_{n<\omega}$ a sequence of closed subsets $F_{n}$ of $X$ with $F_{n} \subset \operatorname{dom}\left(\Omega_{n}\right), n<\omega$. Then the relation $\bigcup_{n<\omega} \Omega_{n}$ contains an open subcontinuous relation $\Omega \subset X \times Y$ with $\bigcup_{n<\omega} F_{n} \subset \operatorname{dom}(\Omega)$. If moreover $\bigcup_{n<\omega} F_{n}$ is closed in $X$, then one can take $\Omega$ to be strongly subcontinuous.

Proof. Since $X$ is normal and $\Omega_{n} \subset X \times Y$ is open, there is an open set $U_{n} \subset X$ with $F_{n} \subset U_{n} \subset \overline{U_{n}} \subset \operatorname{dom}\left(\Omega_{n}\right)$. According to Proposition 2.2 , the restricted relation $\Phi_{n}=\Omega_{n} \uparrow U_{n}$ is strongly subcontinuous. Define relations in $X \times \omega$ by $\Psi=\bigcup_{n<\omega} F_{n} \times\{n\}$ and $\Phi=\bigcup_{n<\omega} U_{n} \times\{n\}$. Evidently, $\Psi$ is closed and $\Phi$ is open (see Proposition 2.1). Hence, by Proposition 2.4, $\Phi$ contains an open subcontinuous relation $\Theta \subset X \times \omega$ with $\operatorname{dom}(\Psi) \subset \operatorname{dom}(\Theta)$. The open relation $\Omega=\bigcup_{n<\omega} \Phi_{n} \uparrow \Theta^{-1}(n) \subset \bigcup_{n<\omega} \Phi_{n}$ is now as required.

Indeed, $\bigcup_{n<\omega} F_{n}=\operatorname{dom}(\Psi) \subset \operatorname{dom}(\Omega)$ because $\operatorname{dom}(\Omega)=\operatorname{dom}(\Theta)$. To see that $\Omega$ is subcontinuous, take $p \in \operatorname{dom}(\Omega)$. Then $p \in \operatorname{dom}(\Theta)$, and by subcontinuity of $\Theta$, there exists an open set $W \subset X$ such that $p \in W$ and $K=\Theta[W]$ is a finite subset of $\omega$. Since each relation $\Phi_{k}$ is strongly subcontinuous, there are also open sets $V_{k} \subset X, k \in K$, with $p \in V_{k}$ and $\Phi_{k}\left[V_{k}\right]$ finite. Then $V=\bigcap_{k \in K}\left(W \cap V_{k}\right)$ is an open set containing $p$ such that $\Omega[V] \subset \bigcup_{k \in K} \Phi_{k}\left[V_{k}\right]$ is finite. Finally, if $F=\bigcup_{n<\omega} F_{n}$ is closed in $X$, then $\Omega \uparrow U$ is strongly subcontinuous (by Proposition 2.2), where $U \subset X$ is an open set with $F \subset U \subset \bar{U} \subset \operatorname{dom}(\Omega)$.

3. Open finite-valued relations. Let $\Phi \subset X \times Y$ be a relation which is a usual map $\Phi: X \rightarrow Y$. In this case, the $Y$-cover of $X$ represented by $\Phi$ is pairwise disjoint. Hence, for a discrete $Y$, we have the following special case of Proposition 2.11(c),

Proposition 3.1. Let $X$ be a space, and $Y$ a discrete space. Then a relation $\Phi: X \rightarrow Y$ is a continuous map if and only if $\Phi$ is a (clopen) discrete $Y$-cover of $X$. In particular, $\Phi: X \rightarrow Y$ is continuous if and only if $\Phi \subset X \times Y$ is open (equivalently, closed).

Precisely as in the case of subcontinuous relations, we can extend the property in Proposition 3.1 to partial maps $\Phi \subset X \times Y$. Namely, we shall say that a relation $\Phi \subset X \times Y$ with $\Phi: \operatorname{dom}(\Phi) \rightarrow Y$ (i.e. a partial map) is continuous if the map $\Phi: \operatorname{dom}(\Phi) \rightarrow Y$ is continuous. Thus, for a discrete $Y$, it follows from Proposition 3.1 that a partial map $\Phi \subset X \times Y$ is continuous if and only if it represents a $Y$-family in $X$ which is discrete in itself, i.e. in $\bigcup_{y \in Y} \Phi^{-1}(y)=\operatorname{dom}(\Phi)$. 
For a set $Y$, we will use $\Sigma_{Y}=\{S \subset Y: 1 \leq|S|<\omega\}$ for the collection of all nonempty finite subsets of $Y$, and for $n<\omega$, we set

$$
[Y]^{n}=\left\{S \in \Sigma_{Y}:|S|=n\right\} .
$$

Thus, $[Y]^{0}=\emptyset$ and $\Sigma_{Y}=\bigcup_{n<\omega}[Y]^{n}$. For a discrete $Y$, we will consider $\Sigma_{Y}$ endowed with discrete topology, or equivalently, with the Vietoris topology generated by the topology of $Y$. Moreover, to each relation $\Phi \subset X \times Y$ we will associate the relation $\Sigma_{(\Phi, Y)} \subset X \times \Sigma_{Y}$ defined by

$$
\Sigma_{(\Phi, Y)}=\left\{\langle x, S\rangle: x \in X \text { and } S \in \Sigma_{\Phi(x)}\right\} .
$$

In other words, $\Sigma_{(\Phi, Y)}$ is defined by $\Sigma_{(\Phi, Y)}(x)=\Sigma_{\Phi(x)}$ for every $x \in X$. Whenever $S \in \Sigma_{Y}$ and $V \subset X$, we find that $V \times S \subset \Phi$ if and only if $V \times\{S\} \subset \Sigma_{(\Phi, Y)}$. Accordingly, the construction preserves open relations.

Proposition 3.2. Let $X$ be a space, and $Y$ a discrete space. Then a relation $\Phi \subset X \times Y$ is open if and only if so is $\Sigma_{(\Phi, Y)} \subset X \times \Sigma_{Y}$.

Let $\Phi: X \rightsquigarrow Y$ be a finite-valued relation. Then it can be regarded as a usual map $\Phi: X \rightarrow \Sigma_{Y}$ from $X$ to $\Sigma_{Y}$. While $\Phi: X \rightsquigarrow Y$ and $\Phi: X \rightarrow \Sigma_{Y}$ are essentially the same relation, their properties may differ depending on the interpretation of the range of the relation. In view of Proposition 3.1, we will be mainly interested in the case when $\Phi: X \rightarrow \Sigma_{Y}$ is continuous. To regard the continuity of $\Phi: X \rightarrow \Sigma_{Y}$ as a property of the relation $\Phi: X \rightsquigarrow Y$, we shall say that $\Phi$ is Vietoris continuous whenever $\Phi: X \rightarrow \Sigma_{Y}$ is continuous. Vietoris continuity is well known in the framework of set-valued mappings, and corresponds precisely to the case when $\Phi: X \rightsquigarrow Y$ is both lower and upper semicontinuous. However, this will play no role in this paper.

In what follows, the preimage of $S \in \Sigma_{Y}$ by $\Phi$ when the relation $\Phi$ is considered as a usual map from $X$ to $\Sigma_{Y}$, will be denoted by

$$
\Phi^{\leftarrow}(S)=\{x \in X: \Phi(x)=S\} .
$$

Proposition 3.3. Let $X$ be a space, $Y$ a discrete space, and $\Phi: X \rightsquigarrow Y$ a finite-valued relation. If $\Phi$ is Vietoris continuous, then it is both clopen and subcontinuous. Conversely, if $\Phi$ is open in $X \times Y$ and $\Phi: X \rightarrow[Y]^{n}$ for some $n \geq 1$, then it is also Vietoris continuous.

Proof. If $\Phi$ is Vietoris continuous and $S \in \Sigma_{Y}$, then $V=\Phi^{\leftarrow}(S)$ is a clopen set in $X$ with $V \times S \subset \Phi$ and $\Phi[V]=S$. This shows that $\Phi$ is both clopen and subcontinuous. Suppose that $\Phi: X \rightarrow[Y]^{n}$ for some $n \geq 1$, and take an $S \in[Y]^{n}$. Then $\Phi^{\leftarrow}(S)=\bigcap_{y \in S} \Phi^{-1}(y)$, which shows that $\Phi$ is Vietoris continuous provided it is open.

The above simple observation implies the following natural representation of finite-valued open relations. 
Proposition 3.4. Let $Z$ be a space, $Y$ a discrete space, and $\Phi: Z \rightsquigarrow Y$ a finite-valued open relation. Whenever $n<\omega$, set

$$
Z_{n}=\left\{z \in Z: \Phi(z) \in[Y]^{n}\right\} \text { and } \Phi_{n}=\Phi\left\lceil Z_{n} .\right.
$$

Then for each $n \geq 1$, the relation $\Phi_{n}: Z_{n} \rightsquigarrow Y$ is Vietoris continuous, while the set $\bigcup_{k \leq n} Z_{k}$ is closed.

Proof. The Vietoris continuity of $\Phi_{n}$ follows from Propositions 3.1 and 3.3 because the relation $\Phi_{n}=\Phi \cap Z_{n} \times Y$ remains open in $Z_{n} \times Y$. If $p \notin \bigcup_{k \leq n} Z_{k}$, then the set $S=\Phi(p)$ contains more than $n$ elements. Since $\Phi$ is open and $S$ is finite, $V=\bigcap_{y \in S} \Phi^{-1}(y)$ is an open set which contains $p$ and satisfies $V \cap \bigcap_{k \leq n} Z_{k}=\emptyset$.

4. Collectionwise normality. In terms of indexed families, a $T_{1}$-space $X$ is collectionwise normal if for every discrete collection $\{\mathscr{F}(y): y \in Y\}$ of closed subsets of $X$ there exists a pairwise disjoint collection $\{\mathscr{U}(y): y \in Y\}$ of open subsets of $X$ such that $\mathscr{F}(y) \subset \mathscr{U}(y), y \in Y$. Regarding these families as relations, collectionwise normality of $X$ can be restated as the property that for every discrete space $Y$ and closed $A \subset X$, every continuous partial map $\Psi: A \rightarrow Y$ is contained in a continuous partial map $\Phi: U \rightarrow Y$ for some open $U \subset X$ with $A \subset U$ (see Propositions 2.1 and 3.1). In other words, $\Phi \uparrow A=\Psi$, and therefore $\Phi$ is an extension of $\Psi$. This is summarised in the proposition below where the extension is subject to a "selection" property with respect to an open relation.

Proposition 4.1. Let $X$ be a collectionwise normal space, $A \subset X$ a closed subset, $Y$ a discrete space, and $\Psi: A \rightarrow Y$ a continuous map. If $\Phi \subset X \times Y$ is an open relation with $\Psi \subset \Phi$, then there exists an open set $U \subset X$ with $A \subset U$, and a continuous map $\Omega: U \rightarrow Y$ such that $\Psi \subset \Omega \subset \Phi$.

Proof. As per the above interpretation of collectionwise normality of $X$, the map $\Psi: A \rightarrow Y$ can be extended to a continuous map $\Theta: V \rightarrow Y$ for some open set $V \subset X$ with $A \subset V$. By Proposition 3.1. $\Theta$ is an open relation in $X \times Y$ because $V \subset X$ is open, and evidently $\Theta\lceil A=\Psi \subset \Phi$. Then $\Omega=\Theta \cap \Phi$ and $U=\operatorname{dom}(\Omega)$ are as required.

We are now ready to prove Theorem 1.1, in fact the following equivalent property in terms of relations.

TheOrem 4.2. Let $X$ be a collectionwise normal space, $A \subset X$ a closed subset, and $Y$ a discrete space. Then for each open relation $\Phi \subset X \times Y$ such that $A \subset \operatorname{dom}(\Phi)$ and $\Phi \uparrow A$ is finite-valued, there exists an open strongly subcontinuous relation $\Omega \subset X \times Y$ with $A \subset \operatorname{dom}(\Omega)$ and $\Omega \subset \Phi$.

Proof. Let $\Theta=\Phi \mid A: A \rightarrow \Sigma_{Y}$. According to Proposition 3.2, the relation $\Sigma_{(\Phi, Y)}$ is open in $X \times \Sigma_{Y}$, and evidently $\Theta \subset \Sigma_{(\Phi, Y)}$. Whenever 
$1 \leq n<\omega$, set

$$
Z_{n}=\left\{x \in A: \Theta(x) \in[Y]^{n}\right\}, \quad \Theta_{n}=\Theta\left\lceil Z_{n}, \quad A_{n}=\bigcup_{k \leq n} Z_{k} .\right.
$$

By Proposition 3.4, each $A_{n}$ is closed in $X$ and each relation $\Theta_{n}: Z_{n} \rightsquigarrow Y$ is Vietoris continuous. For convenience, set $F_{0}=\emptyset=\Omega_{0}$ and $\Psi_{1}=\Theta_{1}$. Since $F_{1}=Z_{1}=A_{1}$ is closed in $X$, by collectionwise normality of $X$ and Proposition 4.1 (applied to the discrete space $\Sigma_{Y}$ ) there exists an open set $U_{1} \subset X$ with $F_{1} \subset U_{1}$, and a continuous map $\Omega_{1}: U_{1} \rightarrow \Sigma_{Y}$ such that $\Psi_{1} \subset \Omega_{1} \subset \Sigma_{(\Phi, Y)}$. Considering $\Omega_{1}$ as a relation in $X \times Y$, it is Vietoris continuous, and $\Omega_{1} \subset \Phi$ because $\Omega_{1} \subset \Sigma_{(\Phi, Y)}$. Set $F_{2}=Z_{2} \backslash U_{1}$, which is closed in $X$ because $Z_{1}=F_{1} \subset U_{1}=\operatorname{dom}\left(\Omega_{1}\right)$, and therefore $F_{2}=A_{2} \backslash U_{1}$. Since $\Psi_{2}=\Theta_{2}\left\lceil F_{2}: F_{2} \rightarrow[Y]^{2}\right.$ is continuous, just as before, $\Psi_{2} \subset \Omega_{2}$ for some open subcontinuous relation $\Omega_{2} \subset \Phi$.

Extending these arguments in an obvious manner by induction, we get a sequence of open subcontinuous relations $\Omega_{n} \subset \Phi$ in $X \times Y$ and a sequence of closed sets $F_{n} \subset X$ such that $F_{n} \subset \operatorname{dom}\left(\Omega_{n}\right)$ and $A=\bigcup_{n<\omega} F_{n}$. We can now apply Corollary 2.5 to get the required relation $\Omega \subset X \times Y$.

In conclusion, let us remark that Theorem 4.2 implies the following natural property, to be used in the next section.

Corollary 4.3. Let $X$ be a collectionwise normal space, $A \subset X$ a closed subset, and $Y$ a discrete space. Then for each open finite-valued relation $\Phi: A \rightsquigarrow Y$ there exists an open subcontinuous relation $\Omega: X \rightsquigarrow Y$ such that $\Omega \uparrow A \subset \Phi$.

Proof. Let $\tilde{\Phi}=\Phi \cup(X \backslash A) \times Y$. By Theorem 4.2, $\tilde{\Phi}$ contains an open strongly subcontinuous relation $\tilde{\Omega} \subset X \times Y$ with $A \subset \operatorname{dom}(\tilde{\Omega})$. The relation $\Omega=\tilde{\Omega} \cup(X \backslash A) \times\left\{y_{A}\right\}$, where $y_{A} \in Y$ is any fixed point, is as required.

5. Dowker's extension theorem. The extension property of collectionwise normality stated in Proposition 4.1 is valid for any Banach range, and is known as Dowker's extension theorem [2].

Theorem 5.1 ([2]). Let $X$ be a collectionwise normal space, and $E$ be a Banach space. If $A \subset X$ is closed, then every continuous $g: A \rightarrow E$ can be extended to a continuous map $f: X \rightarrow E$.

Here, we present a simple proof of this theorem based on an idea in [5]. To this end, we shall say that a partition of unity $\left\{\xi_{y}: y \in Y\right\}$ on a space $X$ is subordinated to a relation $\Omega: X \rightsquigarrow Y$ if $\xi_{y}(x) \neq 0$ implies $y \in \Omega(x)$, equivalently $\xi_{y}^{-1}((0,1]) \subset \Omega^{-1}(y)$.

We now have the following two simple constructions of continuous maps in normed spaces. 
Lemma 5.2. Let $X$ be a collectionwise normal space, $(E,\|\cdot\|)$ a normed space, $A \subset X$ a closed subset, and $g: A \rightarrow E$ a continuous map. If $\varepsilon>0$, then there exists a continuous map $f_{\varepsilon}: X \rightarrow E$ such that $\left\|f_{\varepsilon}(x)-g(x)\right\|<\varepsilon$ for every $x \in A$.

Proof. Since $E$ is paracompact (being metrizable), there exists a locally finite open cover $\mathscr{U}$ of $E$ with $\operatorname{diam}(U)<\varepsilon$ for every $U \in \mathscr{U}$. By taking points $y_{U} \in U, U \in \mathscr{U}$, represent this cover as an open subcontinuous relation $\Phi: E \rightsquigarrow Y$ for some discrete subset $Y \subset E$. Then the composite relation $\Phi \circ g: A \rightsquigarrow Y$ is also open and subcontinuous because $g$ is continuous. Hence, by Corollary 4.3 , there exists an open subcontinuous relation $\Omega$ : $X \rightsquigarrow Y$ with $\Omega \uparrow A \subset \Phi \circ g$. Since $\Omega$ represents a locally finite open cover of the normal space $X$, there exists a partition of unity $\left\{\xi_{y}: y \in Y\right\}$ on $X$ subordinated to $\Omega$. Finally, define the required $f_{\varepsilon}: X \rightarrow E$ by letting $f_{\varepsilon}(x)=\sum_{y \in Y} \xi_{y}(x) \cdot y$ for $x \in X$. Since the partition of unity is locally finite being subordinated to $\Omega$, the map $f_{\varepsilon}$ is continuous.

To show that it is $\varepsilon$-close to $g$ on $A$, pick $p \in A$. Then $y \in \Omega(p) \subset \Phi(g(p))$ implies that $g(p) \in \Phi^{-1}(y)$, and therefore $\|y-g(p)\|<\varepsilon$. Since $\Omega(p)$ is finite because so is $\Phi(g(p))$, we finally get

$$
\begin{aligned}
\left\|f_{\varepsilon}(p)-g(p)\right\| & =\left\|\sum_{y \in \Omega(p)} \xi_{y}(p) \cdot y-\sum_{y \in \Omega(p)} \xi_{y}(p) \cdot g(p)\right\| \\
& \leq \sum_{y \in \Omega(p)} \xi_{y}(p) \cdot\|y-g(p)\|<\sum_{y \in \Omega(p)} \xi_{y}(p) \cdot \varepsilon=\varepsilon .
\end{aligned}
$$

LEMMA 5.3. Let $X$ be a collectionwise normal space, $(E,\|\cdot\|)$ a normed space, $A \subset X$ a closed subset, and $g: A \rightarrow E$ a continuous map. Also, let $\varepsilon>0$ and let $f_{\varepsilon}: X \rightarrow Y$ be a continuous map such that $\left\|f_{\varepsilon}(x)-g(x)\right\|<\varepsilon$ for every $x \in A$. If $\delta>0$, then there is a continuous map $f_{\delta}: X \rightarrow E$ such that

(a) $\left\|f_{\delta}(x)-g(x)\right\|<\delta$ for every $x \in A$;

(b) $\left\|f_{\delta}(x)-f_{\varepsilon}(x)\right\|<\delta+\varepsilon$ for every $x \in X$.

Proof. By Lemma 5.2, there exists a continuous map $f: X \rightarrow E$ such that $\|f(x)-g(x)\|<\delta$ for every $x \in A$. Accordingly, $\left\|f(x)-f_{\varepsilon}(x)\right\|<\delta+\varepsilon$ for every $x \in A$. As in [5, Claim 2.1], consider the set

$$
B=\left\{x \in X:\left\|f(x)-f_{\varepsilon}(x)\right\| \geq \delta+\varepsilon\right\},
$$

which is closed and disjoint from $A$. So, $A \subset \xi^{-1}(0)$ and $B \subset \xi^{-1}(1)$, for some continuous function $\xi: X \rightarrow[0,1]$. Then define the required $f_{\delta}: X \rightarrow E$ by $f_{\delta}(x)=(1-\xi(x)) \cdot f(x)+\xi(x) \cdot f_{\varepsilon}(x)$ for $x \in X$. Evidently, $f_{\delta}\lceil A=f\lceil A$, so (a) holds because it is true for $f$. Similarly, $f_{\delta}\left\lceil B=f_{\varepsilon} \mid B\right.$ and (b) is trivial for the points of $B$. Finally, for $x \notin A \cup B$, 5.1 implies that $\left\|f(x)-f_{\varepsilon}(x)\right\|<\delta+\varepsilon$. 
Therefore,

$$
\begin{aligned}
\left\|f_{\delta}(x)-f_{\varepsilon}(x)\right\| & =(1-\xi(x)) \cdot\left\|f(x)-f_{\varepsilon}(x)\right\| \\
& <(1-\xi(x)) \cdot(\delta+\varepsilon)<\varepsilon+\delta .
\end{aligned}
$$

Proof of Theorem 5.1. Inductively, applying Lemmas 5.2 and 5.3 , for each $n<\omega$ there exists a continuous map $f_{n}: X \rightarrow E$ such that

$$
\begin{aligned}
\left\|f_{n}(x)-g(x)\right\| & <2^{-n} \quad \text { for every } x \in A, \\
\left\|f_{n+1}(x)-f_{n}(x)\right\|<2^{-n+1} & \text { for every } x \in X .
\end{aligned}
$$

Since $E$ is complete (being Banach), by (5.3) the sequence $\left\{f_{n}\right\}$ is uniformly convergent to a continuous map $f: X \rightarrow E$. It is now evident from (5.2) that $f\lceil A=g$.

\section{References}

[1] R. H. Bing, Metrization of topological spaces, Canad. J. Math. 3 (1951), 175-186.

[2] C. H. Dowker, On a theorem of Hanner, Ark. Mat. 2 (1952), 307-313.

[3] R. V. Fuller, Relations among continuous and various non-continuous functions, Pacific J. Math. 25 (1968), 495-509.

[4] V. Gutev, Closed graph multi-selections, Fund. Math. 211 (2011), 85-99.

[5] V. Gutev and N. R. Loufouma Makala, Selections, extensions and collectionwise normality, J. Math. Anal. Appl. 368 (2010), 573-577.

[6] E. Michael, A note on paracompact spaces, Proc. Amer. Math. Soc. 4 (1953), 831838.

[7] E. Michael, Point-finite and locally finite coverings, Canad. J. Math. 7 (1955), 275279.

[8] K. Nagami, Paracompactness and strong screenability, Nagoya Math. J. 8 (1955), 83-88.

[9] S. I. Nedev, Four theorems of E. Michael on continuous selections, B"lgar. Akad. Nauk Izv. Mat. Inst. 15 (1974), 389-393.

[10] S. I. Nedev, Selection and factorization theorems for set-valued mappings, Serdica 6 (1980), 291-317.

[11] T. Przymusiński, Collectionwise normality and absolute retracts, Fund. Math. 98 (1978), 61-73.

[12] R. E. Smithson, Subcontinuity for multifunctions, Pacific J. Math. 61 (1975), 283288.

Valentin Gutev

Department of Mathematics

Faculty of Science

University of Malta

Msida MSD 2080, Malta

E-mail: valentin.gutev@um.edu.mt 
